\title{
EXPERIMENTAL EVALUATION OF THE EFFECTIVENESS OF ONLINE WATER-WASHING IN GAS TURBINE COMPRESSORS
}

\author{
Klaus Brun, Ph.D. \\ Program Director \\ Southwest Research Institute ${ }^{\circledR}$ \\ San Antonio, TX 78228, USA \\ Terrence A. Grimley \\ Manager \\ Southwest Research Institute ${ }^{\circledR}$ \\ San Antonio, TX 78228, USA
}

\author{
William C. Foiles \\ Rotating Equipment Specialist \\ BP Exploration \& Production \\ Houston, TX 77079, USA \\ Rainer Kurz, Ph.D. \\ Manager \\ Solar Turbines, Inc. \\ San Diego, CA 92123, USA
}

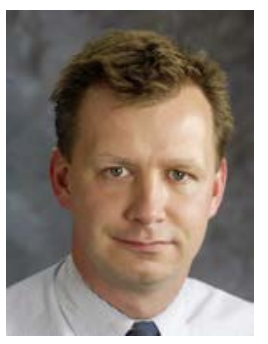

Dr. Klaus Brun is the Program Director of the Machinery Program at Southwest Research Institute. His experience includes positions in engineering, project management, and management at Solar Turbines, General Electric, and Alstom. He holds four patents, authored over 100 papers, and published a textbook on gas turbines. Dr. Brun won an R\&D 100 award in 2007 for his Semi-Active Valve invention and ASME Oil Gas Committee Best Paper awards in 1998, 2000, 2005, 2009, 2010, and 2012. He was chosen to the " 40 under 40 " by the San Antonio Business Journal. He is the chair of the ASMEIGTI Board of Directors and the past Chairman of the ASME Oil \& Gas Applications Committee. He is also a member of the API 616 Task Forces, the Fan Conference Advisory Committee, and the Latin American Turbomachinery Conference Advisory Committee. Dr. Brun is an editor of Global Gas Turbine News, Executive Correspondent of Turbomachinery International Magazine, and an Associate Editor of the ASME Journal of Gas Turbines for Power.

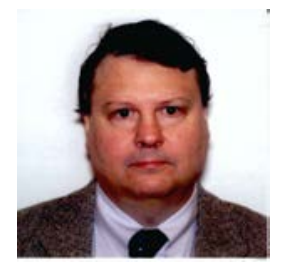

William C. Foiles is a rotating equipment engineer with BP. Prior to BP, he worked in power generation for GE and Babcock and Wilcox, oil and gas with Saudi Aramco, and consulting with GE-Bently Nevada. Mr. Foiles graduated from the Rotating Machinery and Controls (ROMAC) program of the University of Virginia.

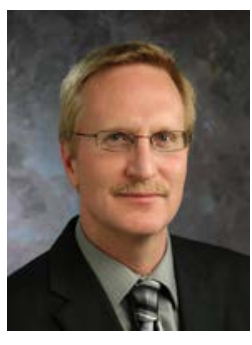

Terrence A. Grimley is the Manager of Southwest Research Institute's Metering Research Facility. He was responsible for the construction and integration of key elements of the weigh tank proving system, development of the data acquisition systems and data reduction software, and commissioning/debugging of the facility. Mr. Grimley has conducted extensive research in the area of multi-path transit-time ultrasonic flow meters. A large portion of the ultrasonic meter research has centered on the effects of upstream piping disturbances and the application of flow conditioners to ultrasonic meter installations. He has also participated in research related to the application of a wide variety of gas flow measurement technologies as well as other experimental fluid mechanics programs.

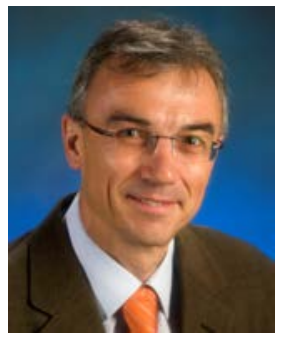

Dr. Rainer Kurz is the Manager of Systems Analysis at Solar Turbines, Inc. in San Diego, California. His organization is responsible for analyzing compression requirements, predicting compressor and gas turbine performance, for conducting application studies, and for field performance testing. Dr. Kurz attended the Universitaet der Bundeswehr in Hamburg, Germany, where he received the degree of a Dr.-Ing. in 1991. He has authored numerous publications about turbomachinery related topics, is an ASME fellow, and a member of the Turbomachinery Symposium Advisory Committee.

\section{ABSTRACT}

An investigation of the effectiveness of online combustion turbine axial compressor washing using various purity grade waters and commercial washing detergents was performed. For this project, blade surface fouling dirt was obtained from gas turbine axial compressor blades installed at various field sites. The dirt was analyzed to determine the composition and consistency of typical blade surface fouling materials. A representative dirt formula and blade coating procedure was developed so that comparative tests could be performed using various cleaning fluids. Dirt coated blades were installed in a wind tunnel capable of simulating compressor operating conditions. A spray nozzle upstream of the blade test section was used for washing blades with five different test liquids to determine the effectiveness or advantages of any liquid. Once this testing was completed, a similar test setup was then utilized to inject a mixture of formulated fouling dirt, and the various 
online cleaning liquids upstream of the blade into the wind tunnel to assess redeposit characteristics. The effect of highpurity water versus regular water on fouling dirt was also studied in separate residue experiments. Results indicate that spraying cleaning fluid into a flowing air stream is a viable means of cleaning a compressor blade. Each of the fluids was able to clean the test blade at both low and high air velocities and at different blade incident angles. Within the parameters/fluids tested, the results indicate that:

1. The blade cleaning is primarily a mechanical function and does not depend on the type of fluid used for cleaning. The results showed that most of the cleaning occurs shortly after the cleaning fluid is introduced into the flow stream.

2. Dirt removed from the blades may redeposit in other areas as the cleaning fluid is evaporated. Redeposit occurred in flow recirculation zones during the cleaning tests, and heated flow tests demonstrated dirt deposit in the presence of a cleaning fluid. In addition, the type of fluid used for cleaning has no effect on the redeposit characteristics of the dirt.

3. Blade erosion was not found to be a significant issue for the short durations that online water-washing was performed. However, uncontrolled water-washing (or overspray) for extended periods of time did result in measureable leading and trailing edge blade erosions.

\section{INTRODUCTION}

Gas turbine axial compressor blades foul due to the deposit of dirt particles during operation. This fouling significantly affects the gas compressor's aerodynamic performance and efficiency, thus forcing the operator to regularly shut down the unit for offline water-washing of the compressor. Alternatively, online washing technologies have been developed to clean the compressor during operation to minimize gas turbine shutdowns and optimize availability. To gain an improved understanding of the effectiveness of online cleaning technologies, specifically the dirt removal and redeposit processes, a number of tests of fouled blades mounted in a high-speed wind tunnel were performed. These tests quantified both the washing effectiveness using various washing fluids as well as the redeposit of foulant dirt in downstream stages.

Some past studies have looked at compressor waterwashing effectiveness. A good summary of past work can be found in Boyce, et al. (2005). Some relevant studies are discussed below:

a Meher-Homji, et al. (2009) found that fouling deterioration of gas turbine primarily depends on two factors: the susceptibility of the engine to compressor fouling based on its design parameters and the sensitivity of the gas turbine to a certain degree of imposed fouling. Similarly, the analysis of gas turbine compressor fouling related with online wash was studied by Vigueras Zuniga (2007) using an experimental test rig. The experimental result demonstrated that the process of washing was assumed to recover the output power until 99.5 percent.
- Hovland and Antoine (2004) proposed the optimization of online and offline compressor washing considering economic benefits with Model Predictive Control model. Schneider, et al. (2009) presented the determination of optimal point between online and offline washing through optimization of industrial gas turbine compressor washing schedule.

口 The research by Ogbonnaya (2011) showed that the compressor efficiency and the overall operational efficiency were improved to 82 percent and 45.8 percent with optimized online water-washing. However, frequent compressor blade washing behaviors can increase the operational and maintenance (O\&M) costs.

Similarly, fouling has been studied in the past by various authors. The mechanisms that lead to the entrainment of particles are, at least theoretically, well understood. However, the conditions under which particles on impact actually stick to the surface of the blades are less clear. Understanding can be gained from the study of fouled blades.

The adherence of small particles to airfoils (i.e., the fouling of their surfaces) will cause a performance deterioration of these airfoils. The deterioration in this case is usually reversible, as the particles can be removed through waterwashing (Kurz and Brun, 2009). This distinction is important because the economic implications of recoverable and nonrecoverable degradation have different economic impacts:

- Fouling can be removed by offline water-washing and slowed down by online water-washing. Theoretically, the engine can be kept at a very small degradation level at all times, if it is frequently washed online, and the cost (i.e., lost production) of shutting the engine down for waterwashing (typically half a day) is carried. The decision to shut the engine down for offline washing is a balance between lost production due to the lower power versus the lost production for shutting the engine down for a certain amount of time.

- The reversal of nonrecoverable degradation requires the engine to be overhauled. Therefore, operators likely will allow much larger levels of nonrecoverable degradation before they take action. Although theoretical considerations (Tarabrin, et al., 1998) indicate that smaller engines show more fouling than bigger engines, there is no anecdotal evidence for this to be true. If there is an impact of engine size, it is probably obscured by the fact that smaller engines usually have fewer compressor stages, and, more importantly, by the exact circumstances of site dust load and air filtration system.

Meher-Homji, et al. (2009) introduced the important distinction between susceptibility and sensitivity to fouling. Susceptibility is the amount of fouling a compressor incurs under a specified contaminant load, while sensitivity describes the effect on compressor efficiency—or, in a wider sense, gas turbine power output capability and efficiency—of a certain amount of compressor fouling. The paper does not address the other more permanent consequence of particle ingestion, that is, the potential for hot corrosion as a result of salt particles 
entering the engine and reacting with sulfur from fuel or combustion air.

To assess the fouling mechanisms, observed fouling patterns must be analyzed. Findings from both the literature and from observations recorded are discussed. Vigueras Zuniga (2007) reports deposits on the gas turbine compressor rotor and vanes, with deposits both on suction and pressure surface. There is evidence of increased deposits in the leading edge region of the rotor blade suction side. The deposits exist on both the suction and pressure side of the rotor blades, with fewer deposits near the leading edge and in the hub region. The deposits seem to indicate the location of the transition area of the boundary layer.

Parker and Lee (1972) studied fouling patterns on rotating blades for very fine $(0.13$ to $0.19 \mathrm{~lm})$ particles. Sample results of the estimated deposition rates for different regions of the blade surface were reviewed. These results show high deposition rates at the blade leading edge, relatively low deposition on the pressure side, and a higher deposition rate on the suction side toward the trailing edge. The deposition rates on the suction surface near the trailing edge are where the boundary layer is thick and turbulent.

On the other hand, Syverud, et al. (2007) detected in a gas turbine subjected to salt water spray deposits mainly on the blade pressure side and the blade leading edges, causing a significant increase in surface roughness. They also found, like other researchers, that the majority of the deposits occurred on the early stages of the compressor. It must be noted that the salt water spray in these experiments formed larger size and wet droplets (23 lm median volume diameter). Obviously, the relative humidity (and with it the salt particle size) drops in the latter compressor stages due to the temperature increase. Typical particle sizes after air filters in industrial gas turbines will be much lower. As a result of the acceleration of the inlet air when it enters the compressor through a bellmouth and inlet guide vanes, the relative humidity of the air will increase. An ambient relative humidity of 50 percent can, therefore, lead to condensation at the inlet guide vanes. The droplets that can form due to this effect may scrub entrained solids, such as salts, as well as some gases like $\mathrm{CO}_{2}$ or $\mathrm{SO}_{x}$. Because they form downstream of the filter, their droplet size can be larger than the particle sizes normally prevented from passing through the air filter. They also will create an acid atmosphere within the compressor; thus, causing corrosion pitting on the blades (corrosion pitting can be prevented by appropriate coatings).

Another area that is affected by fouling is the compressor shroud or casing. Elrod and Bettner (1983) compared the performance of the axial compressor of a gas turbine for different shroud roughness levels. Comparing the results for design roughness $(1.8 \mathrm{~lm})$ with a rough $(13 \mathrm{~lm})$ shroud, the compressor loses about 1 percent in flow capacity and about 1 percent in peak efficiency. The added wall roughness increases the wall boundary layer displacement thickness.

The type of foulants entering the compressor varies widely from site to site. Deposits of oil and grease are commonly found in industrial locations as a result of local emissions from refineries and petrochemical plants, or from internal lube oil leaks (Meher-Homji, et al., 2009). These types of deposits act as "glue" and entrap other materials entering the compressor. Lube oil ingested into the flow path is spread by centrifugal and aerodynamic forces and generates a film on ten blades that allows even larger particles to stick to the surface.

Coastal locations usually involve the ingestion of sea salt, desert regions attract dry sand and dust particles, and a variety of fertilizer chemicals may be ingested in agricultural areas.

The fouling deposit mechanisms were discussed in detail by Kurz and Brun (2012) and are, thus, not further discussed herein. Similarly, the impact of fouling on gas turbine performance has been discussed in many papers and a good summary can be found in Kurz and Brun (2009).

\section{PROJECT SCOPE}

Within the project, fouling dirt was sampled from 12 actual field operation gas turbine axial compressor blades to characterize the composition and consistency of typical blade surface fouling. This dirt was analyzed to determine a chemical composition and physical properties using $\mathrm{x}$-ray diffraction, dispersion spectroscopy, and carbon hydrogen nitrogen sulfur (CHNS) analysis. A representative dirt formula and blade coating procedure was reverse engineered for realistic and repeatable testing of blade cleaning and redeposit in a wind tunnel environment. The formulated dirt was then applied to gas turbine compressor blades at thicknesses observed similar to the field installations. These blades were mounted in a transsonic wind tunnel with an upstream spray nozzle that simulates compressor operating conditions during online washing.

Washing was performed with five different liquids (three grades of water, a commercial compressor cleaning product, and a laundry detergent) to determine the effectiveness or advantages of any of the liquids. Four different flow rates and two different angles of attacks were tested. To determine the cleaning effectiveness, blades were carefully weighted before and after each test to quantify the amount of fouling dirt removed from each blade during the test. Photos of the blade surface were also digitally processed using threshold differentiation techniques to determine localized cleaning effectiveness.

Once this testing was completed, a similar test setup was then utilized to inject a mixture of formulated fouling dirt, and the various online cleaning liquids upstream of the blade into the wind tunnel to assess foulant redeposit characteristics. The effect of high-purity water versus regular water on fouling dirt was also studied in separate residue experiments. Also, a study was undertaken to evaluate the impact of water-washing on blade erosion.

\section{PROJECT OBJECTIVES}

The objective of this project was to provide a thorough experimental evaluation on the effectiveness of online turbine 
cleaning with various cleaning agents, including high-purity deionized (DI) water. The Phase I investigation found that the existing literature contained mostly case studies on online washing and lacked complete scientific evidence on the process of online cleaning and the use of high-purity DI water.

The objectives of this study can be summarized with four questions:

1. Does online cleaning work?

2. Is there any difference between any of the online cleaning liquids?

3. Will dirt be removed during online cleaning redeposit once the cleaning liquid has been evaporated?

4. Do any liquids reduce the redeposit of dirt in the online washing process?

The sections that follow provide a discussion of the methods utilized in this study to address these four questions.

\section{BLADE DIRT ANALYSIS AND SIMULATED DIRT DEVELOPMENT}

An industrial gas turbine manufacturer provided seven sample blades severely fouled and five sample scrapings from a wide range of onshore and offshore locations. These samples were utilized in creating the standard dirt formulation. The resultant dirt composition is intended to provide a mixture representative of the sample locations.

\section{Dirt Development Overview}

To create standard "dirt" representative of materials found in blade deposits operating turbines, sample dirty turbine blades and blade scrapings were analyzed. Specifically, the blades were examined qualitatively for the adhesion properties of the contamination and observations were made regarding the deposit locations and patterns. The next section, Blade Samples, provides a summary of the blade pictures and the observations. The scrapings were analyzed to determine their composition for use in developing a standard formulation.

\section{Blade Samples}

Pictures of representative blade samples are given in Figure 1 through Figure 6. The blades have varying degrees of contamination but have some common characteristics:

a In general, the deposits were primarily on the front (convex) portion of the blade (see pictures of the front and back of Blades 13 and 10).

a The streaking patterns evident on all the blades suggest that the material is deposited via radial flow from the root of the blade out.

a The cleaned area in Figure 1 (Blade 14) shows where a paper towel was rubbed lightly on the blade to remove the material. The dirt does not appear to adhere tightly to the blades. A small amount of force is all that was required to dislodge the material.

- In several cases, the leading edge of the blade was cleaner that the rest of the blade. This suggests that areas with high velocity and incident angle are less susceptible to dirt deposit; however, other blades suggest that potential separation areas are less susceptible to having the dirt stick.

a Some of the deposits, like that shown for Blade 9, appear to have a substantial amount of hydrocarbon mixed in with the "dirt."

Based on these blades, a decision was made to coat only the convex portion of the blades used for testing.

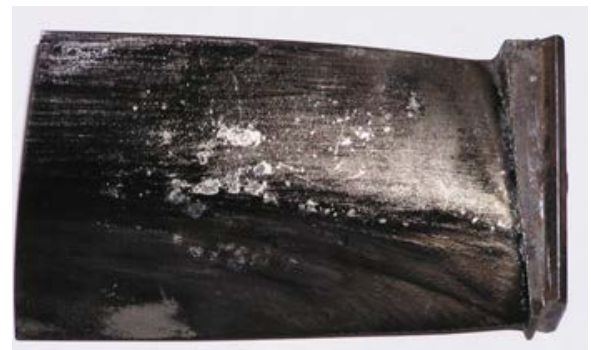

Figure 1. Blade Sample 14

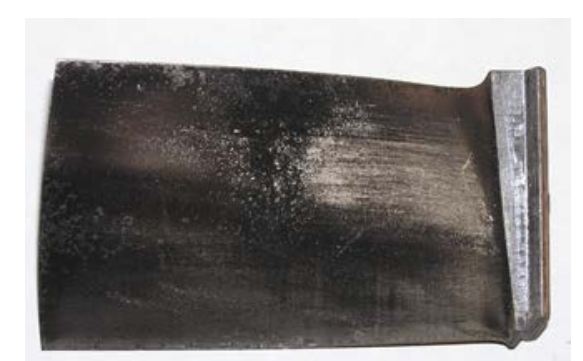

Figure 2. "Front” Side of Blade Sample 13

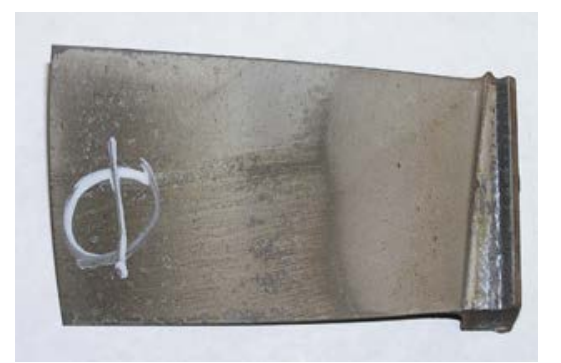

Figure 3. "Back” Side of Blade Sample 13

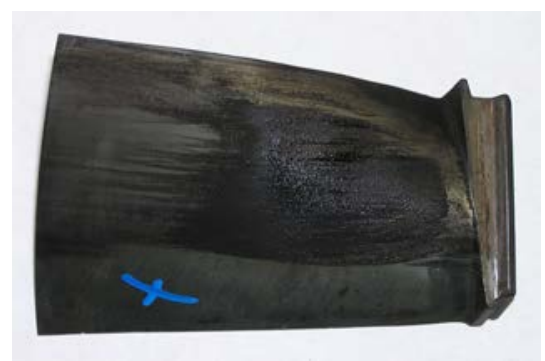

Figure 4. Blade Sample 9 


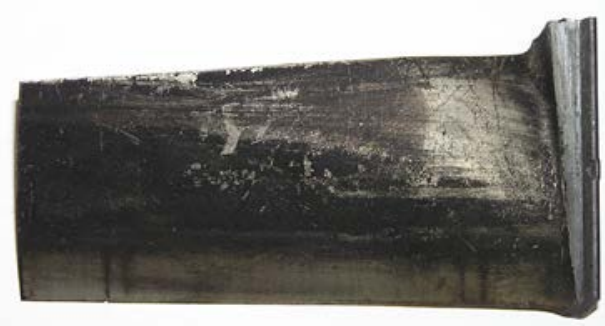

Figure 5. "Front” Side of Blade Sample 10

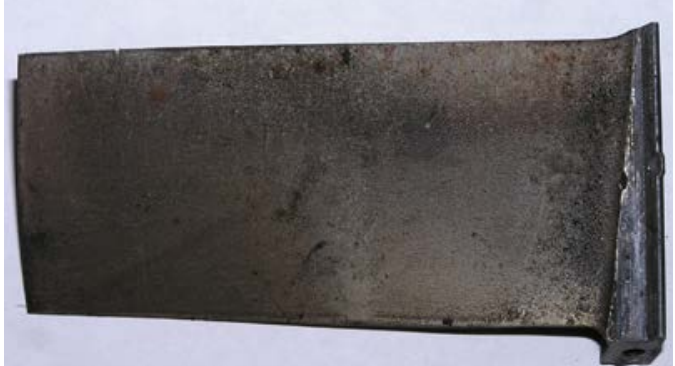

Figure 6. “Back” Side of Blade Sample 10

\section{Sample Dirt Analysis}

Chemical analyses performed on turbine "dirt" samples included x-ray diffraction (XRD), x-ray dispersion spectroscopy (EDS), and carbon hydrogen nitrogen sulfur analysis (CHNS - performed on Sample 1 only). EDS reveals relative amounts of elements present that are heavier than oxygen. XRD identifies crystalline compounds in the sample. CHNS is a combustion process that provides the total amounts of carbon, hydrogen, nitrogen, and sulfur only. The XRD identified several different compounds, both major and minor contributors. Most of the major contributing compounds were included in the simulated dirt admixture, but many minor components were excluded to simplify the mixture and avoid excessive materials cost. These minor components contained ions and groups common to some major components, so the resulting formulation would not be significantly different. Examples of excluded compounds included $\mathrm{CaSiO}_{3}, \mathrm{Ca}_{2} \mathrm{SiO}_{4}$, $\mathrm{CaSiO}_{3}$, and $\mathrm{CaSO}_{4} \bullet 2 \mathrm{H}_{2} \mathrm{O}$.

\section{$X$-Ray Diffraction}

X-Ray Diffraction (XRD) was used to determine what compounds are present in the "dirt" samples. EDS and CHNS provide elemental data, but do not tell how these atoms are arranged in compounds. XRD produces a spectrum with peaks indicative of crystalline compounds, which are identified only qualitatively by the instrument software. The software provides only a broad "suggestion" as to the amount present of each compound; compounds are labeled as "major" or "minor" contributors. Amorphous compounds are not identified, but the total amount of amorphous components can be estimated if it is a large contribution. A very broad peak appears in the baseline, and the area underneath can be used to estimate percent amorphous compounds. The estimated amorphous portion is about 80 percent. From the color and texture of the sample, it was apparent that the majority of this portion was carbon.
Sample 4: $\quad \mathrm{NaCl}$ Major

$\mathrm{Na}_{2} \mathrm{SO}_{4}$ Major

$(\mathrm{Mg}, \mathrm{Al})_{3}(\mathrm{Si}, \mathrm{Al})_{2} \mathrm{O}_{5}(\mathrm{OH})_{4}$ Minor

$\mathrm{MgSiO}_{3}$ Major

Sample 5: $\quad \mathrm{CaSO}_{4} \bullet 2 \mathrm{H}_{2} \mathrm{O}$ Minor

Al Minor

$\mathrm{KAl}\left(\mathrm{SO}_{4}\right)_{2} \bullet 12 \mathrm{H}_{2} \mathrm{O}$ Major

\section{XRD Results}

\begin{tabular}{ll}
\hline Sample 1: & $\left(\mathrm{NH}_{4}\right)_{2} \mathrm{SO}_{4}$ Major \\
& $\mathrm{KAl}\left(\mathrm{SO}_{4}\right)_{2} \bullet 12 \mathrm{H}_{2} \mathrm{O}$ Minor
\end{tabular}


$\mathrm{CaCO}_{3}$ Minor

$\mathrm{SiO}_{2}$ Minor

Sample 7: $\quad \mathrm{CaSiO}_{3}$ Major

$\mathrm{CaCO}_{3}$ Major

$\mathrm{Ca}_{2} \mathrm{SiO}_{4}$ Major

$\mathrm{Na}_{2} \mathrm{SO}_{4}$ Major

Sample 12: No XRD data

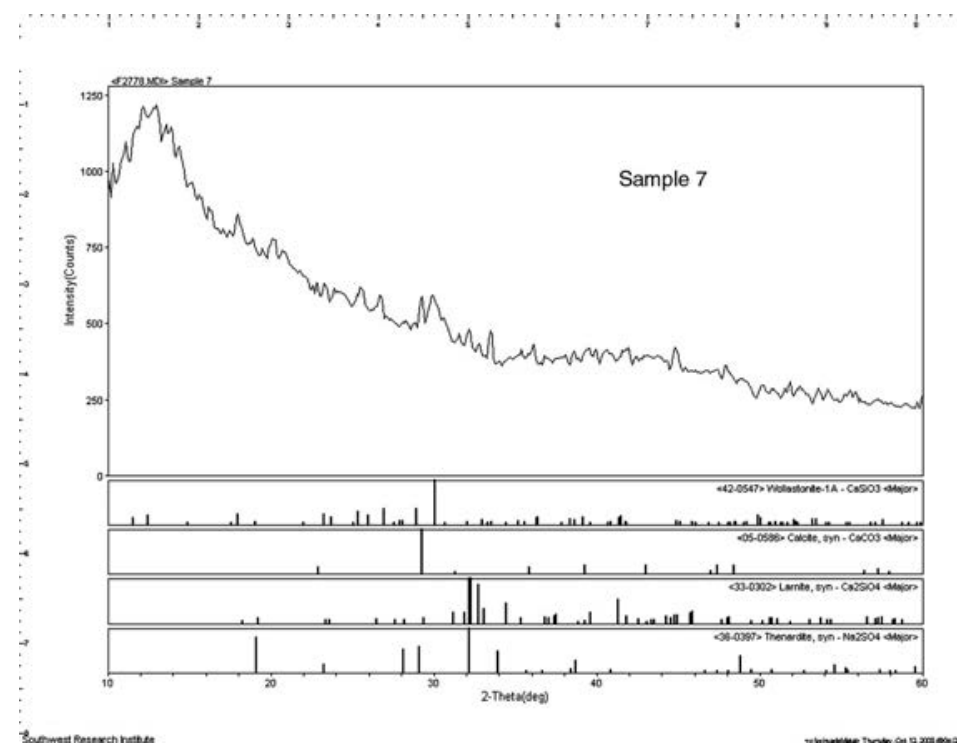

Figure 7. Example XRD Spectrum for Sample 7

\section{X-Ray Dispersion Spectroscopy and Carbon Hydrogen} Nitrogen Sulfur (CHNS) Analysis

X-Ray Dispersion Spectroscopy (EDS) identifies elements with atomic mass units greater than 23 ( $\mathrm{Na}$, sodium) (and oxygen with lesser accuracy) and their percent contribution to the sample. However, the percent reported is overstated, if there is a high contribution from elements of low atomic mass $(\mathrm{H}, \mathrm{C}$, $\mathrm{N})$. The percentages reported were from all elements detected, not of all elements actually present. In the EDS data, only elements from oxygen up (mass-wise) were listed, yet the total percent was 100 percent. Of course, this was not representative of the entire sample since most of the sample was carbon, and carbon was not included in the listing. The numbers must be considered as percentages of the sample excluding carbon. For example, if the sample is 40 percent carbon, then all percentages of elements in the EDS data are multiplied by 0.6 to get the "true" percent contribution of each.

XRD and EDS showed that no two of these five samples were identical, and sometimes the numbers differed greatly. Of all the elements included in the mix, the raw data numbers and averages from EDS are shown below. One must recall that this is of the non-carbon containing portion of the sample, so actual percentages are lower by at least one-half. The largest differences between samples are 1.8 percent sodium for Sample 1 and 14.7 percent sodium for Sample 4; 1.4 percent silicon in Sample 4 but 37 percent in Sample 5; no chlorine in Sample 12 but 20 percent in Sample 4. 
Table 1. EDS Data for Major Elements Included in Simulated "Dirt"

\begin{tabular}{|c|c|c|r|r|c|c|}
\hline Sample & Na & Mg & \multicolumn{1}{c|}{ Si } & \multicolumn{1}{c|}{ S } & Cl & Ca \\
\hline 1 & 1.82 & 1.30 & 4.06 & 31.9 & 1.52 & 1.05 \\
\hline 4 & 14.65 & 5.29 & 1.35 & 21.78 & 20.49 & 1.52 \\
\hline 5 & 2.06 & 1.46 & 36.92 & 14.68 & 1.70 & 2.78 \\
\hline 7 & 4.87 & 2.01 & 4.69 & 15.34 & 5.30 & 0.00 \\
\hline 12 & 4.92 & 0.00 & 7.61 & 14.93 & 0.00 & 0.97 \\
\hline Average & 5.66 & 2.01 & 10.93 & 19.73 & 5.80 & 1.26 \\
\hline
\end{tabular}

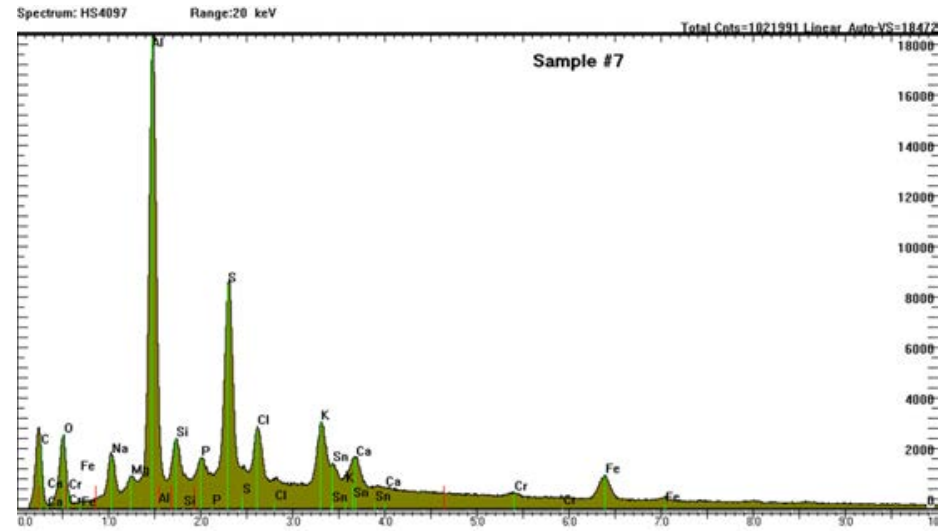

Figure 8. Example EDS Results for Sample 7

As for the other light elements not detected by EDS, helium is too light to be present. It is noble gas and would not likely be found within a compound. Beryllium, lithium, and boron are not likely to be present, and do not show up with $\mathrm{XRD}$. Hydrogen is so light it never makes up a significant percent of total weight for a mixture like this, and the hydrogen component of the simulated "dirt" is probably larger than the original samples due to the addition of PEG. Nitrogen and carbon are detected by CHNS.

Table 2. CHNS Results on Sample 1

\begin{tabular}{|l|c|c|c|c|}
\hline \multicolumn{1}{|c|}{ Sample ID } & $\begin{array}{c}\text { Conc. } \\
\text { \% C }\end{array}$ & $\begin{array}{c}\text { Conc. } \\
\text { \%H }\end{array}$ & $\begin{array}{c}\text { Conc. } \\
\text { \% N }\end{array}$ & $\begin{array}{c}\text { Conc. } \\
\text { \% S }\end{array}$ \\
\hline Sample 1 & 40.63 & 4.12 & 3.97 & 5.83 \\
\hline Sample 1 Duplicate & 39.83 & 4.19 & 4.09 & 5.94 \\
\hline
\end{tabular}

Carbon content for Sample 1 is found to be 40 percent; however, the baseline in the XRD data shows an amorphous component around 80 percent. Amorphous silica was considered to be another contributor to the amorphous portion, but EDS found only 11 percent silicon on average, and that is just a percentage of the non-carbon contributors. The true percent silicon would be no more than half of that. It appears that carbon makes up more than 40 percent of the amorphous portion of the sample, on average. Since the simulated dirt was intended to be representative of the sample dirt compounds, and not necessarily a rigorous match of the samples, the decision was made to include 50 percent carbon lampblack in the formula.

As for the nitrogen, XRD revealed that only Sample 1 contained any nitrogen at all, so it was not considered in formulating the admixture.

\section{Standard Dirt Formulation}

Combining data from multiple instruments like this to reverse engineer a formula always reveals conflicts in the data. EDS shows an average of 19.7 percent sulfur of the non-carbon portion of the samples. Since it was estimated that 50 percent of the sample is carbon, this suggests that 9.85 percent of the entire sample was sulfur. XRD says sulfur is present solely in the form of the sulfate ion, $\mathrm{SO}_{4}{ }^{2-}$, which is present in the major contributor $\mathrm{Na}_{2} \mathrm{SO}_{4}$. To include sulfur at 9.85 percent, then 43.7 percent of the entire sample would have to be $\mathrm{Na}_{2} \mathrm{SO}_{4}$. However, this number is much too large. That would mean every other component would have to be reduced to a total of $100-50-43.7=6.3$ percent. This is obviously wrong when considering the contribution of other elements to the whole, according to EDS and XRD. Therefore, the CHNS data, which is considered more accurate than EDS, was used to determine the sulfur level at about 5.9 percent. This results in 26.1 percent $\mathrm{Na}_{2} \mathrm{SO}_{4}$ in the simulated dirt, which appears to be more reasonable.

The rest of the major element percentages were taken from the EDS data and multiplied by 0.6 to get values closer to the real values. Chlorine, magnesium, silicon, and calcium are listed in Table 3 with their percent contributions. For each element, the percentages were divided by their atomic mass and multiplied by the formula mass of the compound they are found in to get the percent contribution to the whole of each compound. Since $\mathrm{MgSiO}_{3}$ already provided some silicon, that amount of silicon was subtracted from the calculation of $\mathrm{SiO}_{2}$.

Amorphous carbon content was suggested by XRD for all samples to be as high as 80 percent, but CHNS results listed only 40 percent carbon for Sample 1. A 50 percent average contribution from carbon was approximated for the admixture. EDS was performed on all samples $(1,4,5,7$, and 12) to determine the amount of each component that should be added. Percentages of major contributing elements were averaged over all samples. Using compound formula weights, calculations were done to determine how much of each should be added to the admixture to achieve elemental percentages determined by EDS. Table 3 shows average percent elemental contributions, and percent of corresponding compounds added to the batch.

Table 3. Percent of Elemental Components in "Dirt" Sample and Corresponding Compounds in Admixture

\begin{tabular}{|c|c|}
\hline $\begin{array}{c}\text { Avg. Elemental \% } \\
\text { of Sample }\end{array}$ & $\begin{array}{c}\text { Compound Percent of } \\
\text { Admixture }\end{array}$ \\
\hline \hline $3.5 \% \mathrm{Cl}$ & $5.8 \% \mathrm{NaCl}$ \\
\hline $1.2 \% \mathrm{Mg}$ & $5 \% \mathrm{MgSiO} 3$ \\
\hline $6.6 \% \mathrm{Si}$ & $5 \% \mathrm{MgSiO} 3 \mathrm{\&} 11.2 \% \mathrm{SiO} 2$ \\
\hline $0.76 \% \mathrm{Ca}$ & $1.9 \% \mathrm{CaCO} 3$ \\
\hline $5.9 \% \mathrm{~S}$ & $26.1 \% \mathrm{Na} 2 \mathrm{SO} 4$ \\
\hline $50 \% \mathrm{C}$ & $50 \%$ Carbon Lampblack \\
\hline
\end{tabular}

Example calculation: $100 \%=100 \mathrm{~g}$ for a $100 \mathrm{~g}$ batch $\mathrm{NaCl}$ 
Turbine Blade Coating Formulation

Dry Admixture Components, 100 gram batch (same as percent mass)

$50.0 \mathrm{~g}$ Carbon Lampblack (amorphous carbon)

$5.8 \mathrm{~g} \mathrm{NaCl}$ (sodium chloride, salt)

$26.1 \mathrm{~g} \mathrm{Na}_{2} \mathrm{SO}_{4}$ (sodium sulfate)

$1.9 \mathrm{~g} \mathrm{CaCO}_{3}$ (calcium carbonate)

$11.2 \mathrm{~g} \mathrm{SiO}_{2}$ (silicon IV oxide)

$5.0 \mathrm{~g} \mathrm{MgSiO}_{3}$ (enstatite, magnesium silicate)

\section{Solvent and Binder Mixture Proportion}

$1 \mathrm{~g}$ PEG (poly [ethylene glycol], Average $M_{n}=3400$ )

$20 \mathrm{~mL}$ DCM (dichloromethane)

The PEG was added as a binding agent. Tests with PEG at half the above concentration had poor film characteristics, but with the amount of PEG shown, the film resembled that of the sample turbine blades.

The dichloromethane solvent was used as a carrier so that the mixture could be sprayed onto the blades using a commercial spray guy. The DCM evaporated as the mixture was sprayed.

Dry carbon/salt admixture is mixed with solvent in 1 gram: $20 \mathrm{~mL}$ ratio and is 1:1 with PEG. PEG is dissolved in solvent before combining with the admixture. PEG will remain in solution, but the carbon and other components of the admixture will settle out shortly, therefore, the mixture must be agitated to maintain the dispersion when applying to blade surface.

\section{SINGLE BLADE TEST FACILITY}

A variable-speed, electric-motor-driven centrifugal air compressor (shown in Figure 9) was used to provide flow for the single blade test facility. The compressor suction source was ambient air that was discharged into a 6-inch diameter pipe section. A 19-tube bundle, used to eliminate any swirling flow, was located in the piping upstream of the test section plenum (as shown in Figure 9).
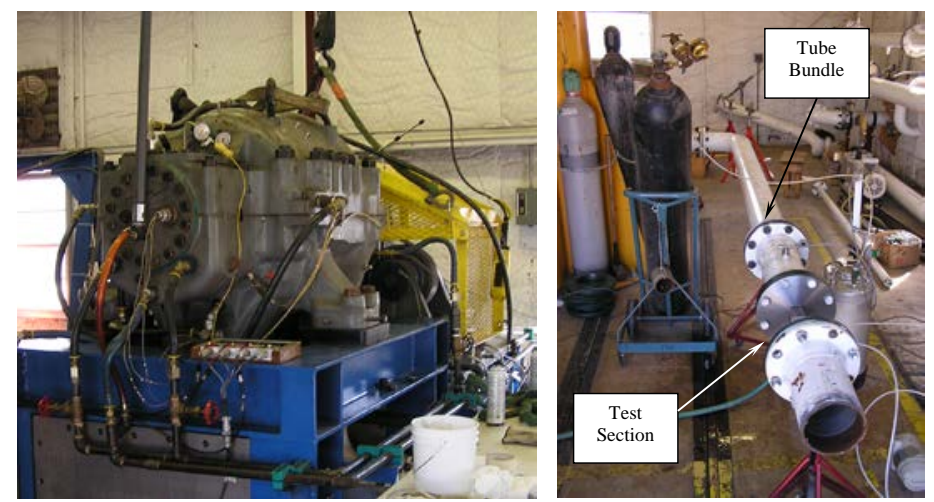

Figure 9. Centrifugal Compressor and Flow Section

\section{Test Section and Blade Mounting}

A test section was designed to allow the test blade to be exposed to a high-velocity air stream to simulate the range of velocities at the inlet to a turbine. The facility was designed to provide a well-developed flow profile in the test section and a
Mach number of up to 0.8. The test section was customdesigned and fabricated to match the $4^{\text {th }}$ stage rotor blade of a mid-size industrial gas turbine. The $4^{\text {th }}$ stage blade was used (as opposed to a $1^{\text {st }}$ stage blade) so that appropriate flow rates could be achieved with existing Southwest Research Institute ${ }^{\circledR}$ flow capabilities. A model of the test section is given in Figure 10. The model shows the contoured inlet section that accelerates the flow from the 6-inch steel pipe plenum into the 1.75 -inch tall by 1.25 -inch wide rectangular section and past the blade. The abrupt change in area of the inlet contour piece was designed to provide a uniform velocity profile at the inlet of the rectangular section. The coned outlet section provides pressure recovery to decrease the total pressure drop requirements on the compressor. The region surrounding the blade was constructed with three clear polycarbonate windows to allow observation of the blade cleaning process. The windows were designed so that they could be easily replaced to maintain a clear view of the test blade.

Five turbine blades were provided for use in these tests. Figure 11 shows one of the blades captured in the blademounting fixture. Note the pins in the bar section; these were used to provide positive position stops to allow the blade to be aligned with the base at 0 degrees relative to the flow, or with the base of the blade at 7 degrees relative to the flow direction. When the blade is installed at the 7-degree angle, the chord at the half-height location of the blade is approximately aligned with the flow direction. The use of the pins allowed for excellent repeatability of the test blade angle.

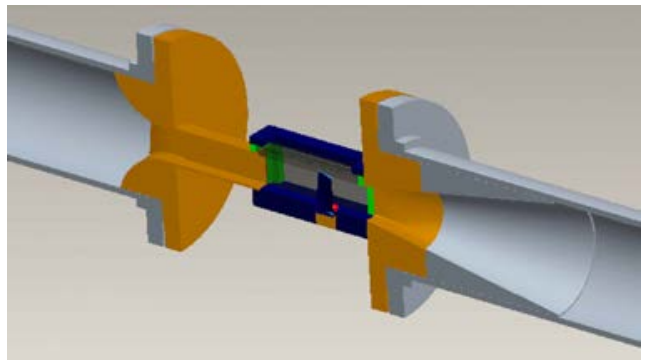

Figure 10. Single Blade Test Section Converging and Diverging Sections

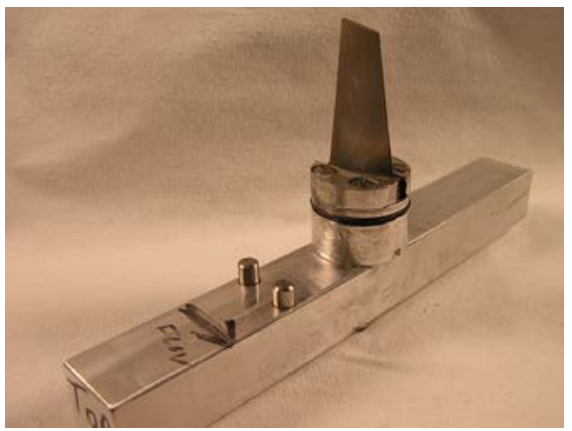

Figure 11. Blade Mounting Fixture with Blade Installed

\section{Flow Rates/Velocities}

Gas turbine axial compressor rotor blades encounter local flow velocities between Mach 0.5 and Mach 0.9. Thus, the local flow velocities in the test section were measured to determine the compressor operating points required to consistently simulate these high subsonic Mach numbers. 
Initial tests were performed with a Kiel probe installed in the test section. The Kiel probe is similar to a Pitot probe in that it provides static to stagnation pressure difference that can be utilized to determine flow velocities from Bernoulli's equation (for subsonic flow). However, the Kiel probe includes a shroud that makes the probe less sensitive to alignment with the flow direction. These tests were used to map the test section velocity as a function of compressor speed and at the blade angles to be used for the cleaning tests. This was necessary because the Kiel probe would not function properly if exposed to the cleaning fluid. Figure 12 shows a top view of the test section with the Kiel probe installed upstream of the turbine blade. Note that the blade alignment fixture was not installed when this picture was taken.

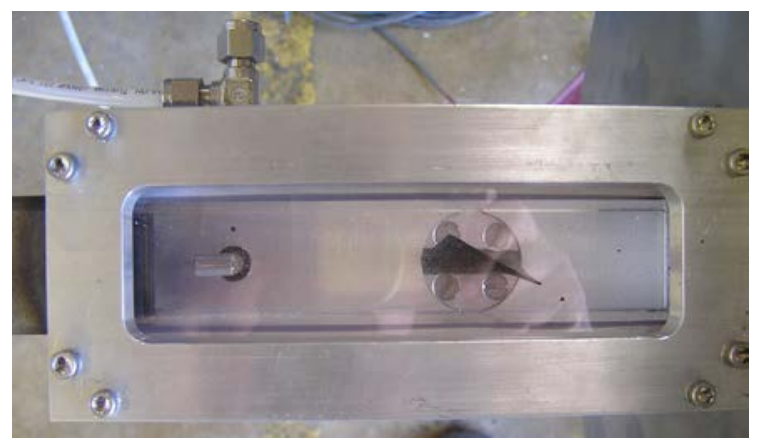

Figure 12. Top View of Blade Mounted in Test Section with Kiel Probe Upstream (Flow is from Left to Right)

Figure 13 shows a curve of the Mach number at the blade as a function of compressor speed. The complete curve is shown for the case when the blade was mounted at 0 degrees and a few test points are shown for the case when the blade was mounted at 7 degrees. The difference in the effective blockage produced by the blade resulted in the different velocities as the blade angle was changed from 0 to 7 degrees. For the 0 degree case, the compressor speeds were chosen at 5,000 rpm and $10,500 \mathrm{rpm}$; these speeds correspond to Mach numbers of approximately 0.3 and 0.6 , respectively. When the blade was positioned at 7 degrees, the corresponding compressor speeds were determined to be 4,800 rpm and 8,500 rpm, respectively.

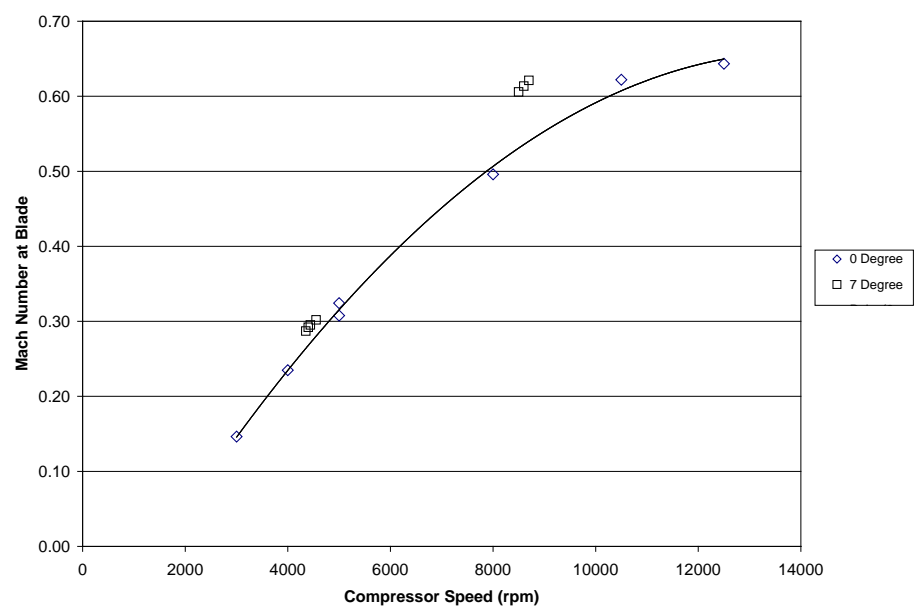

Figure 13. Mach Number at Blade as a Function of Compressor Speed
Fluid Delivery System

A stainless steel tank was pressurized with zero-air to deliver the cleaning fluid to the nozzle as shown in Figure 14. The zero-air was used to avoid any contamination of the highpurity deionized water and the other cleaning fluids. The tank was thoroughly rinsed between each fluid to avoid crosscontamination.

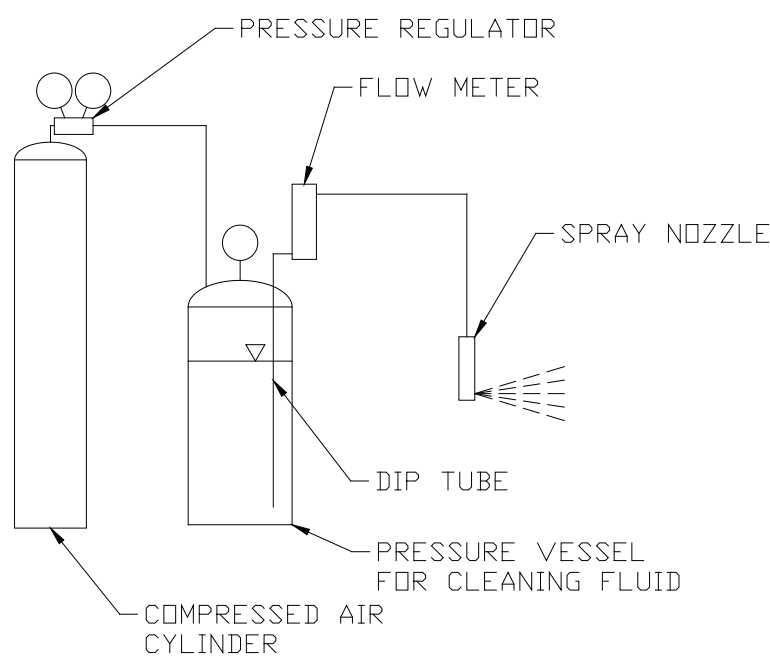

Figure 14. Schematic of Fluid Delivery System

The cleaning fluid was delivered into the plenum upstream of the test section (as shown in Figure 15) using a commercially available cleaning nozzle. The nozzle is reported to produce a cleaning spray with 120-micron droplets when sprayed at 120 psig. Because the nozzle was intended to be used to clean more than a single blade, part of the spray was blocked in order to produce an air-to-water ratio in the range of 200 to 1,000 based on values reported in the literature (Mund and Pilidis, 2004). The mass flow air/water ratio was measured to be roughly 200 for an airflow Mach number of about 0.6. The nozzle and the blockage tube are shown in Figure 16; when mated together, a portion of the spray leaves through the hole in the blockage tube and the remainder of flow travels down the tube.

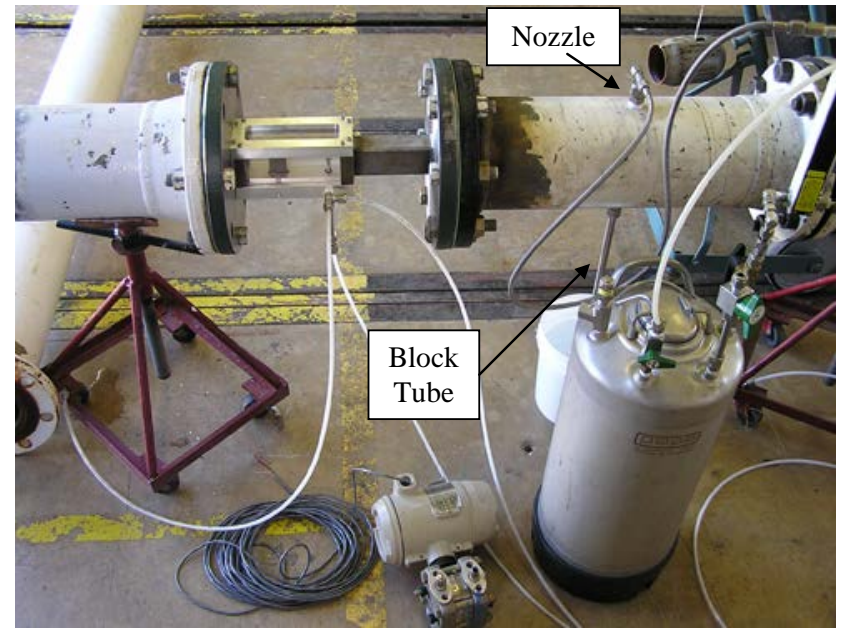

Figure 15. Test Section with Cleaning Fluid Tank and Spray Nozzle Location (Flow is from Right to Left) 


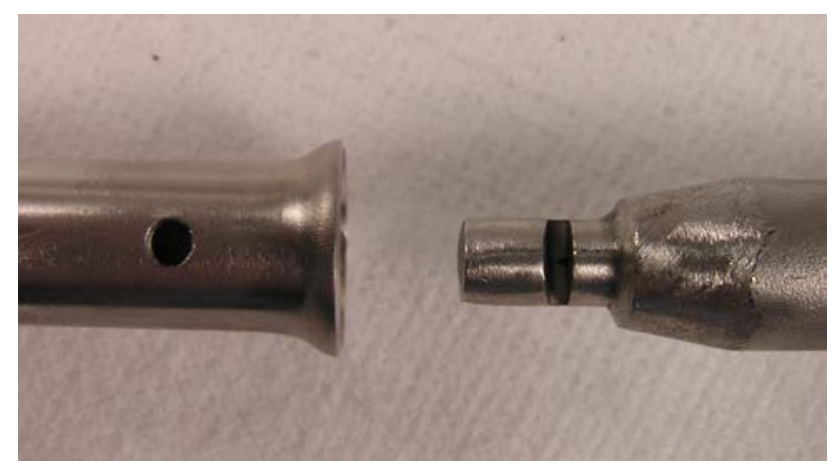

Figure 16. Flow Blockage Tube (Left) and Nozzle (Right)

\section{ONLINE BLADE WASH TESTS}

The sections that follow describe how the blades were cleaned, coated, and flow tested.

\section{Coating Procedure}

The test blades were cleaned with tap water to remove any loose material. The blades were then submerged in a mixture of Simple Green ${ }^{\circledR}$, acetone, and isopropyl alcohol and placed in an ultrasonic cleaning bath for 15 to 20 minutes. This cleaning procedure was repeated until any residual "dirt" had been removed from the blades.

The blades were then wiped with acetone to remove any residual oil/grease from being handled. The blades were weighed prior to mounting in a grooved fixture. The final cleaning prior to coating was performed with a de-fluxing cleaner that leaves no residue.

The blades were sprayed with a standard paint spray gun operating at approximately 35 psi. Twenty passes with the spray gun were made on the row of blades. The blades were then dried under a vapor hood for at least one hour. Finally, the coated blades were re-weighed using the same scale as used for the original measurement.

\section{Blade Coating History}

The plot shown in Figure 17 indicates that amount of dirt material deposited on the blades for testing. The reason for the variation in the deposit amount is not apparent, but tests conducted with DI water at different coating levels indicated that the cleaning level was not a function of the initial coating thickness.

\section{Blade Weight History}

As the blades were weighed multiple times, it was noticed that the blade mass decreased over time. Figure 18 shows a plot of the uncoated blade weight for each time the blade was weighed. The plot shows a consistent loss of material among all blades. However, Blade 5 was only used for some of the blade wash tests; primarily when there was flaking or other problems with one of the other test blades. This suggests that it was the cleaning process used to prepare the blades for coating and/or the coating itself (corrosion) that resulted in the material loss. The surface of the blades became pitted over time. Because of the potential risk to the turbine, the blade mass loss bears further investigation.

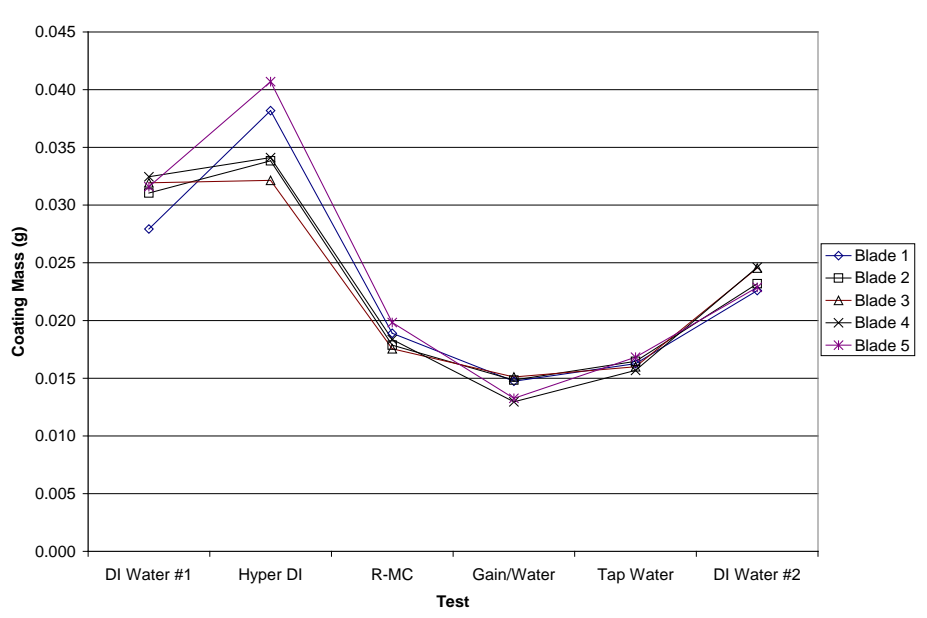

Figure 17. Blade Coating History

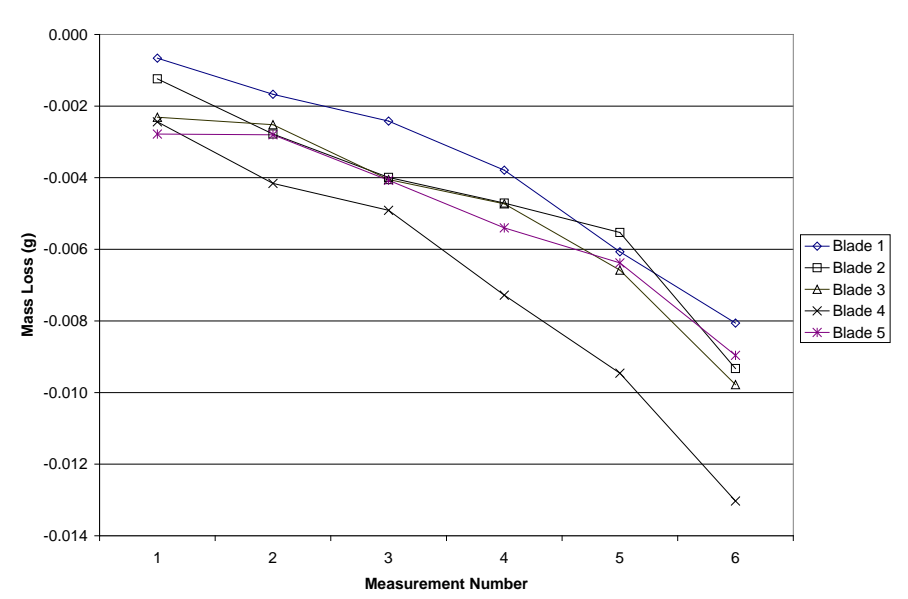

Figure 18. Blade Mass History

\section{Fluids Tested}

Five fluids were used for the blade cleaning tests. Three of the fluids were different "grades" of water: tap water, standard deionized (DI) water, and high-purity deionized water. The ionization levels for the three types of water are given in Table 4.

Table 4. Water Ionization Levels

\begin{tabular}{|l|c|}
\hline \multicolumn{1}{|c|}{ Fluid } & $\begin{array}{c}\text { Resistivity } \\
\text { (M } \mathbf{\Omega}-\mathbf{c m})\end{array}$ \\
\hline Tap Water & $0.002-0.003$ \\
\hline Deionized Water & 10 \\
\hline High-purity Deionized Water & 18 \\
\hline
\end{tabular}

The other two cleaning fluids tested were a commercial compressor cleaning fluid and a 200:1 mixture of deionized water and Gain liquid laundry detergent.

\section{Test Procedure}

The basic sequence followed for testing the different fluids was to first clean and coat the blades as previously described and then to "condition" the blades by flowing air at the test velocity for 10 minutes. The purpose of the "conditioning" activity was to dislodge any loose material prior to exposing the blade to a cleaning solution. Initial tests suggested this was 
necessary to remove poorly bonded "dirt;" however, once the blade cleaning and coating procedure was established, there was no noticeable material loss or flaking during the conditioning time. Blades were photographed after the conditioning runs.

Once all the blades were conditioned, tests were conducted in order from the highest flow rate to the lowest flow rate as shown in Table 5.

Table 5. Test Sequence

\begin{tabular}{|c|c|c|c|}
\hline $\begin{array}{c}\text { Test } \\
\text { Sequence }\end{array}$ & $\begin{array}{c}\text { Compressor } \\
\text { Speed } \\
\text { (rpm) }\end{array}$ & $\begin{array}{c}\text { Mach } \\
\text { Number }\end{array}$ & $\begin{array}{c}\text { Blade } \\
\text { Angle } \\
\text { (degree) }\end{array}$ \\
\hline 1 & 10,500 & 0.6 & 0 \\
\hline 2 & 8,500 & 0.6 & 7 \\
\hline 3 & 5,000 & 0.3 & 0 \\
\hline 4 & 4,800 & 0.3 & 7 \\
\hline
\end{tabular}

Blade 1 was used for test sequence 1; Blade 2 for test sequence 2, etc. Therefore, the same blade was exposed to the same flow conditions for each of the test fluids. On a few occasions, Blade 5 was substituted for another blade, if there was poor adhesion in the original coating or if some other anomaly occurred. Each blade was cleaned for 10 minutes in the flowing gas stream.

After each test, the blade was removed from the test section and photographed. Prior to installing the next blade in the test section, the compressor speed was increased to remove any residual liquid from the upstream plenum and test section.

\section{Analysis Methods}

Digital pictures were used to determine the amount of dirt remaining on the blade at the conclusion of each cleaning test. To ensure repeatable pictures and lighting, a fixture was used to maintain the positions of the lights, diffusers, camera, and the blade.

A public domain image-processing package named "ImageJ" (developed by the U.S. National Institutes of Health) was used for processing the images. The location of the blade in each picture was outlined manually as shown in Figure 19. A macro, developed using the image processing software, was then used to convert and threshold the image to determine the area of the outlined portion and to threshold the image to determine the amount of "dirt" remaining in the area of interest by assessing the total area of the blade and the area contained in the dark regions as shown in Figure 20 and Figure 21. This process was repeated on several independent images of each test result to assess the repeatability of the method.

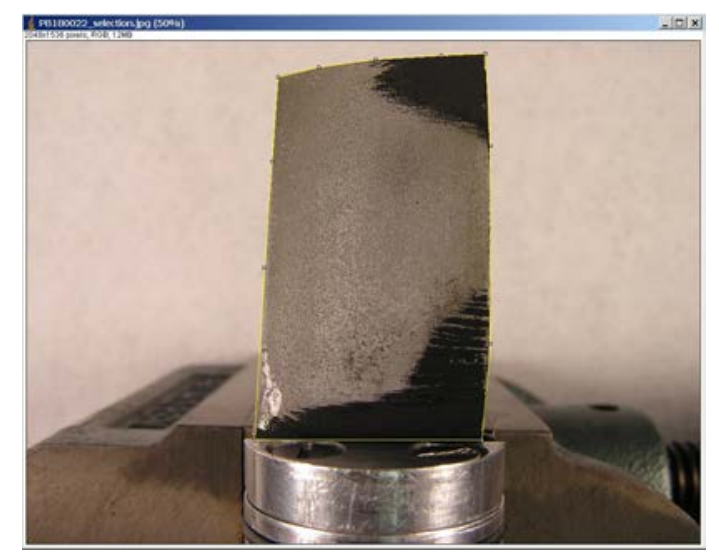

Figure 19. Blade Image Outlined for Processing
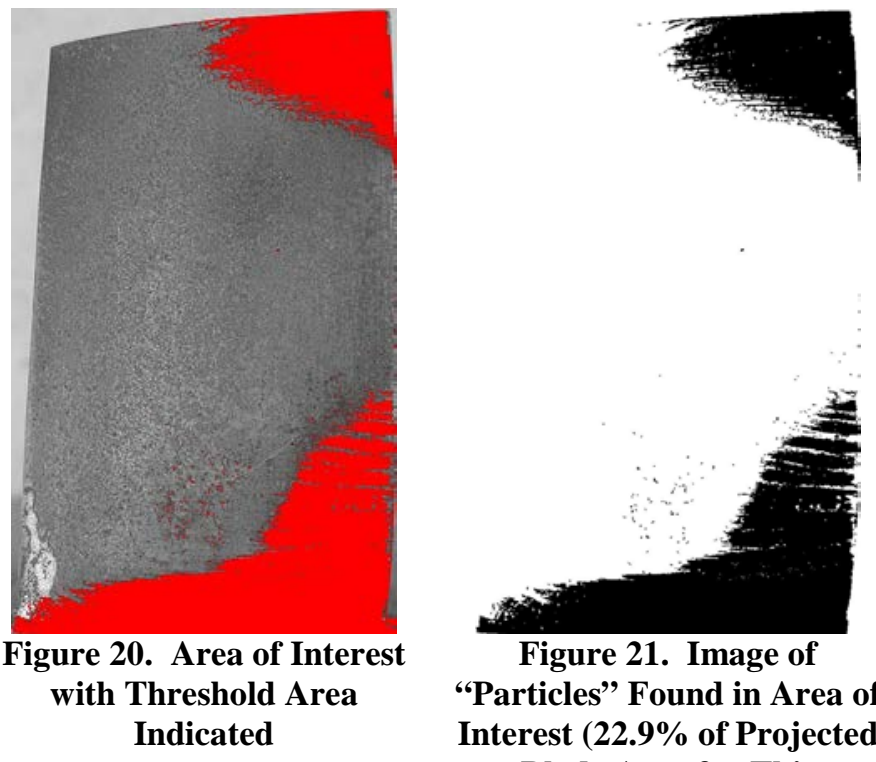

Figure 21. Image of "Particles" Found in Area of Interest (22.9\% of Projected Blade Area for This Example)

\section{Test Results}

Figure 22 and Figure 23 show typical pictures of the front and back of the blades after each cleaning test. These are samples of the pictures used to obtain the results summarized later in this section. Figure 22 shows the case for cleaning with deionized water while Figure 23 shows the commercial detergent washing.

Although the blades were cleaned for 10 minutes each, most of the cleaning occurred within the first few seconds after the liquid spray was initiated. The longer cleaning time allowed liquid adhering to the blade to penetrate onto areas of the blade where there was some flow separation. The streaking of the dirt surface indicated the presence of this mechanism. 


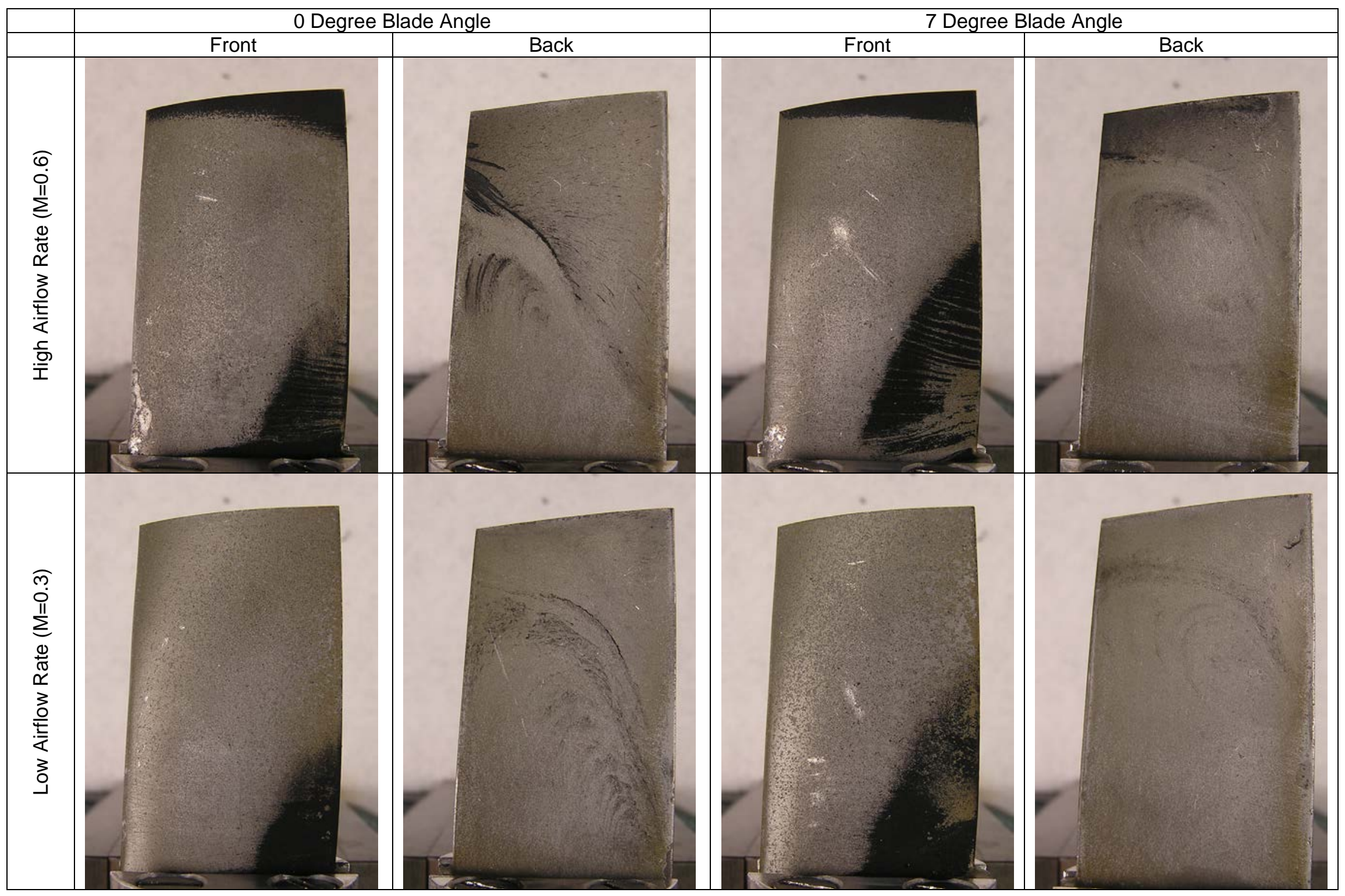

Figure 22. Deionized Water Blade Cleaning Results (Tested Nov. 30) 


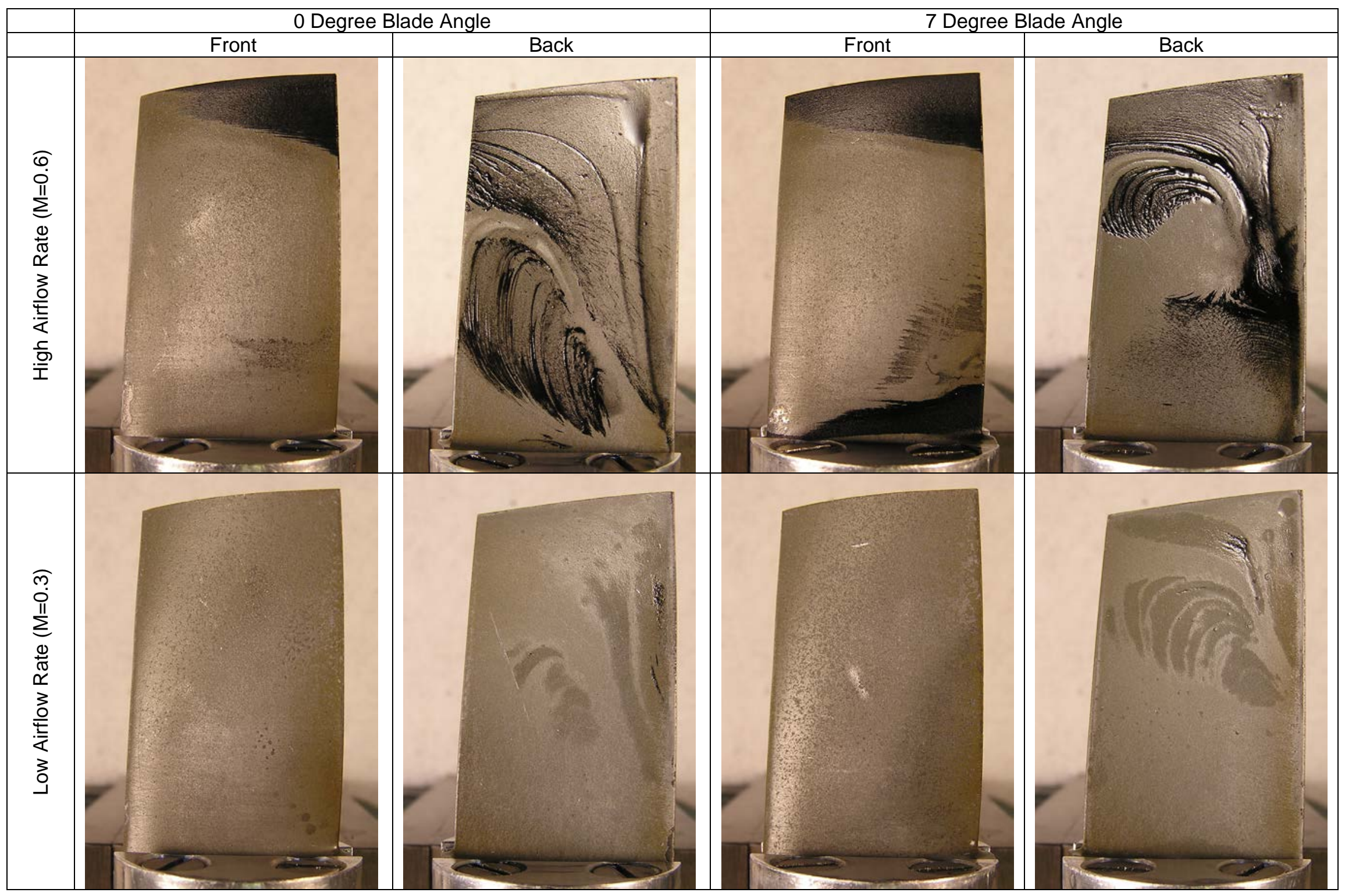

Figure 23. Commercial Detergent Blade Cleaning Results (Tested Nov. 21) 
The residual dirt patterns suggest that cleaning occurs primarily in active flow portions of the blade. Flow recirculation areas on the back of the blade allowed for dirt cleaned from the front of the blade to redeposit on the back of the blade. The flow patterns on a blade installed in a compressor wheel would not have the same patterns because of the presence of the neighboring blades; however, other flow areas within the system may have flow separation zones and these zones are where dirt redeposit would be expected. The primary features of the residual dirt patterns in the figures were independent of the fluid used to clean the blade.

Redeposit on the back portion of the blades was also lower for the low velocity tests. There did not appear to be a significant difference in the amount of redeposit between the different test fluids. However, the commercial detergent cleaning fluid had some foaming during the test and appeared to have some foaming within the redeposit as indicated by the 3-dimensional pattern on the back of the blades shown in Figure 23.

Table 6 summarizes the results of the single-blade cleaning tests. Figure 24 presents the same data in graphical form. The values presented in the table are the average values from analyzing three pictures of the blade (as discussed in Analysis Methods). The uncertainty in measuring the clean area from the image is less than 1 percent. The repeat test data in Table 6 for the DI water and the high-purity DI water suggest that the testto-test repeatability is on the order of 5 percent to 7 percent.

Table 6. Blade Wash Tests: Percent Clean Summary

\begin{tabular}{|c|c|c|c|c|c|}
\hline \multirow{2}{*}{ Date } & \multirow{2}{*}{ Fluid } & \multicolumn{2}{|c|}{ Low Flow Air } & \multicolumn{2}{|c|}{ High Flow Air } \\
\hline & & 0 deg & $7 \mathrm{deg}$ & 0 deg & 7 deg \\
\hline 29-Nov-2005 & Tap Water & $87.2 \%$ & $81.7 \%$ & $79.0 \%$ & $77.6 \%$ \\
\hline 17-Nov-2005 & DI Water \#1 & $96.9 \%$ & $92.8 \%$ & $86.4 \%$ & $74.1 \%$ \\
\hline 30-Nov-2005 & DI Water \#2 & $89.8 \%$ & $83.5 \%$ & $82.8 \%$ & $77.4 \%$ \\
\hline 18-Nov-2005 & $\begin{array}{l}\text { High-purity DI } \\
\text { Water \#1 }\end{array}$ & $94.2 \%$ & $87.3 \%$ & $77.0 \%$ & $79.5 \%$ \\
\hline 18-Nov-2005 & $\begin{array}{c}\text { High-purity DI } \\
\text { Water \#2 }\end{array}$ & $94.3 \%$ & $88.4 \%$ & $67.2 \%$ & $74.7 \%$ \\
\hline 22-Nov-2005 & Gain/DI Water & $99.1 \%$ & $94.2 \%$ & $74.4 \%$ & $79.6 \%$ \\
\hline 21-Nov-2005 & $\begin{array}{c}\text { Commercial } \\
\text { Detergent Wash }\end{array}$ & $99.7 \%$ & $99.5 \%$ & $91.9 \%$ & $84.7 \%$ \\
\hline & Average & $94.5 \%$ & $89.6 \%$ & $79.8 \%$ & $78.2 \%$ \\
\hline & Std Deviation & $5 \%$ & $7 \%$ & $10 \%$ & $5 \%$ \\
\hline
\end{tabular}

The results suggest that there was not a significant difference in the cleaning capability of the fluids tested. Since the percent clean values for the detergents were typically higher than the average, it could be argued that the detergents were more effective; however, the differences were of the same order of magnitude as the test-to-test repeatability. Within the different types of deionized water, the results were again within the test-to-test repeatability. These results, along with the observation that the largest part of the blade cleaning occurs within the first few seconds of liquid application, suggest that the primary means of blade cleaning is through the mechanical removal of the dirt and not through a chemical/solvent process.

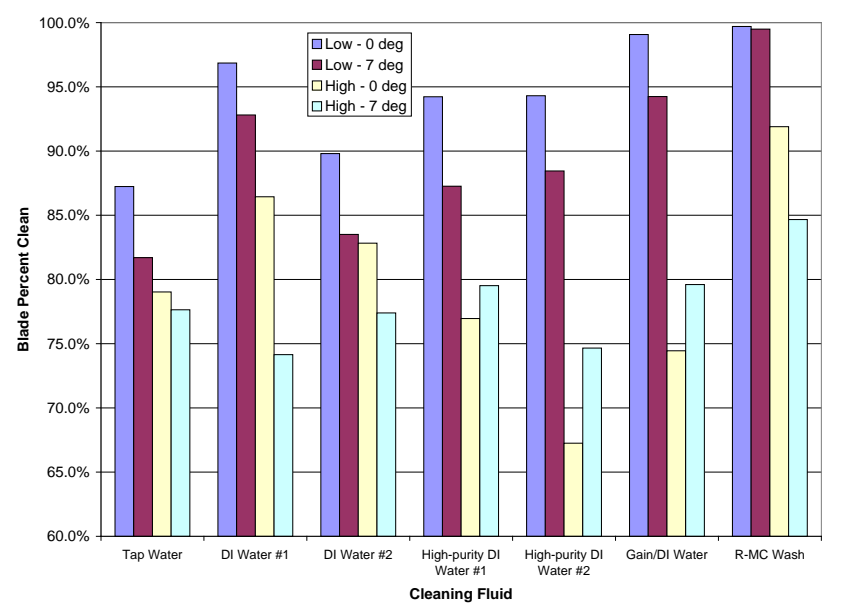

Figure 24. Graphical Summary of Blade Cleaning Results

Within each fluid test, there were consistently better cleaning results for the tests with a 0 degree blade angle than the results for a 7 degree blade angle (i.e., better blade cleaning was evident when the flow separation area on the dirty surface of the blade was decreased). Again, flow separation on the blades is not something that should be expected when there are neighboring blades as there would be on a turbine wheel, but these results suggest that flow separation within the turbine may provide areas for dirt buildup.

There was a difference between the overall results for high and low flow rates; cleaning performed at the low flow rate was consistently more effective. The lower air-to-water mass ratio (about 91 versus 200) present at the lower flow velocity condition may have contributed to the increased cleaning effectiveness present at low velocities.

\section{DIRT REDEPOSIT TESTS}

Particle redeposit on downstream stages of the compressor is particularly important to consider in online washing technology. In an online cleaning program, the later stages of the compressor may be contaminated by redeposition of fouling particles from previous upstream stages or by residual particles from the cleaning solution itself. Though the initial fouling of compressor blades tends to occur in the first few stages, fouling in any stage will affect the compressor efficiency.

Although the specific causes of deposit and/or redeposit are not well understood, one of the potential mechanisms that may allow a dirt particle cleaned from a blade to redeposit occurs when the cleaning solution is evaporated by the temperature rise through the compressor. Once the particle is no longer suspended in the cleaning fluid, there is a higher likelihood of the dirt particle redepositing on a surface.

Three sets of tests were performed to assess differences between the redeposit characteristics of different types of cleaning fluid when the base fluid is evaporated after removing the dirt from the blade. These tests involved different methods of evaporating the carrier fluid to provide insight into the effect of the fluid on dirt redeposit. The sections that follow describe these tests and the results. 


\section{Spray Gun Tests (Heated/Unheated)}

The same paint spray gun used to coat the blades for the cleaning tests was used to assess redeposit of dirt on a clean blade when the dirt was mixed with one of the cleaning fluids. The same proportions of dirt (and PEG) and fluid were mixed into the spray gun; however, for these tests, the fluid used to suspend the dirt was one of the cleaning fluids.

Initial tests with a dirt/deionized water mixture sprayed at ambient temperature showed essentially no dirt deposit on the blade. The presence of the DI water did not allow the dirt to stick to the blade even though the water was atomized by the spray gun.

Additional tests were performed using a low velocity heated air stream produced by flow from a "torpedo" heater as shown in Figure 25. For these tests, the blade was placed 12 inches from the front of the heater, and the spray was directed into the flow stream at an angle (as shown in the figure), such that the air stream could evaporate the liquid from the spray.

Figure 26 through Figure 29 show the pictures of the blades for the heated air stream tests. Although the coating thickness is considerably less than when sprayed using DCM as the carrier fluid, the figures show essentially the same level of

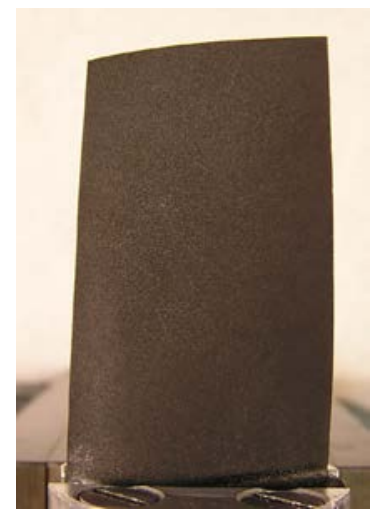

Figure 26. Blade Sprayed with Dirt/Tap Water Mixture in Heated Air Stream

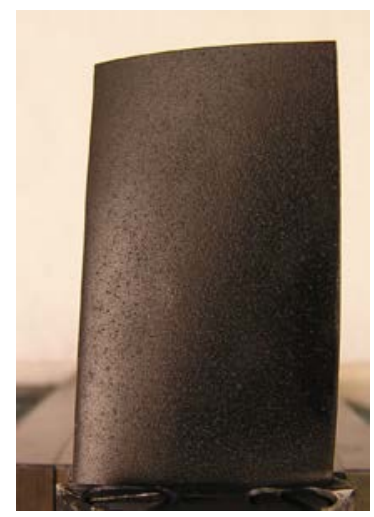

Figure 28. Blade Sprayed with Dirt/Commercial Detergent Mixture in Heated Air Stream deposit for all fluids tested. These tests demonstrated that the redeposit occurs independent of the test fluid used as the carrier. This suggests that exposure to the cleaning fluids used for these tests has no effect on the dirt's deposit mechanism.

\section{Flowing Deposit Tests}

The purpose of these tests was to determine if a mixture of dirt and cleaning fluid would deposit on the blade if the fluid were vaporized in the presence of the dirt.

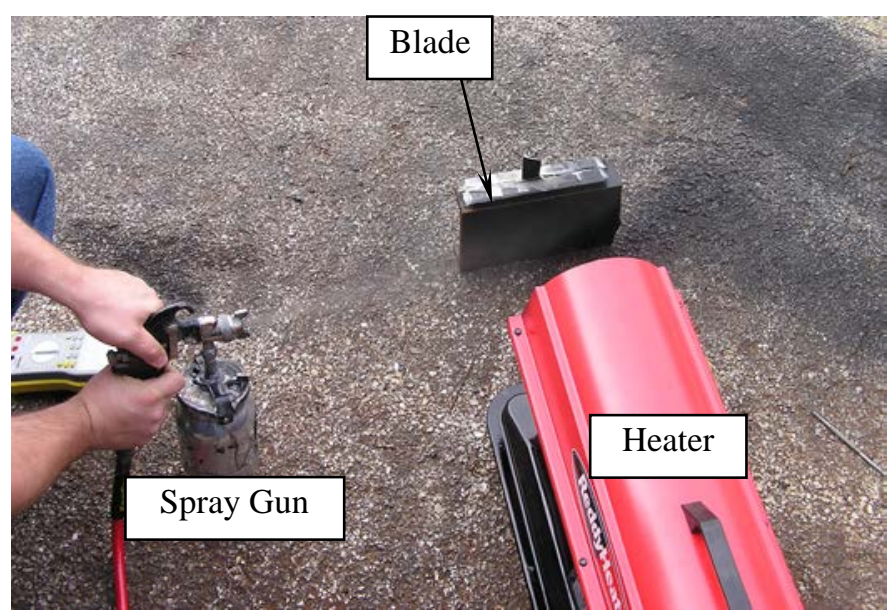

Figure 25. High Temperature Air Spray Tests

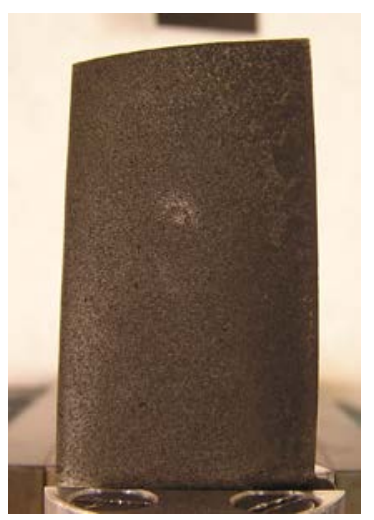

Figure 27. Blade Sprayed with Dirt/High-Purity Deionized Water Mixture in Heated Air Stream

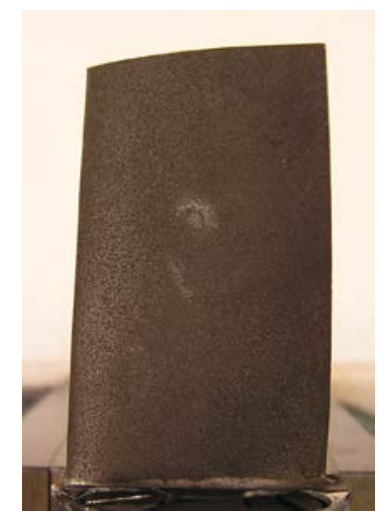

Figure 29. Blade Sprayed with Dirt/Gain/Deionized Water Mixture in Heated Air Stream 
Tests were conducted by heating a mixture of dirt and water and injecting into the plenum upstream of the test section (in the same location used to spray cleaning fluid for the other tests). Clean blades were exposed to the dirt-contaminated water and flowing air stream for a period of about 30 minutes and then photo documented. Tests were conducted using both deionized water and high-purity deionized water and was performed at a very low flow velocity with "room temperature" air as well as at a mid-range velocity with heated air. The specific test conditions are given in Table 7.

Table 7. Flowing Deposit Test Conditions

\begin{tabular}{|c|c|c|}
\hline Test & $\begin{array}{c}\text { Mach No. } \\
\text { at Blade }\end{array}$ & $\begin{array}{c}\text { Air Temperature } \\
\left({ }^{\circ} \mathbf{F}\right)\end{array}$ \\
\hline 1 & $<0.1$ & $60-70$ \\
\hline 2 & 0.38 & 140 to 150 \\
\hline
\end{tabular}

The dirt/water mixture was created using approximately $10 \mathrm{~g}$ of the "standard" dirt/PEG mix per 3,500 $\mathrm{ml}$ of water. This mixture was continuously stirred and was pumped through a copper tube heat exchanger immersed in oil heated in the range of $220^{\circ} \mathrm{F}$ to $290^{\circ} \mathrm{F}$. A schematic of the setup is shown in Figure 30 and a picture of the physical setup is shown in Figure 31. A nozzle with an opening of about 0.032 inches was fabricated for the dirt injection tests. The opening was two to three times the opening size of the cleaning nozzle to avoid clogging the nozzle; however, clogging did occur occasionally during the tests, and the pump had to be stopped and started several times during a test to clear the nozzle and maintain flow.

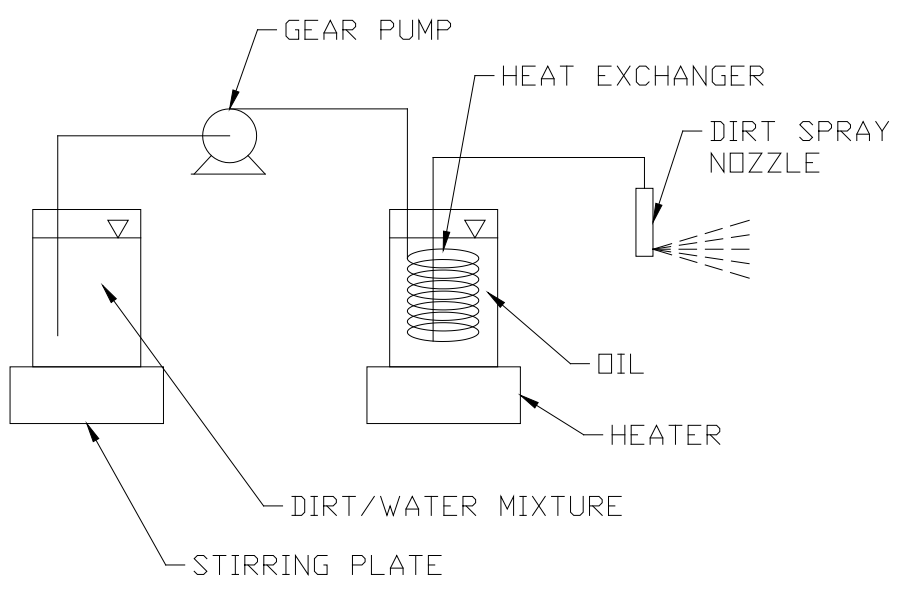

Figure 30. Schematic of Dirt Injection System

Although the intent was to produce water at temperatures above the atmospheric boiling point (by maintaining elevated pressure in the copper tube) that would vaporize once sprayed into the air stream, the system could produce steam only for the first few minutes of operation. While this was not the intended operating mode, it was acceptable for the comparative tests since this mode was consistent for all the tests.

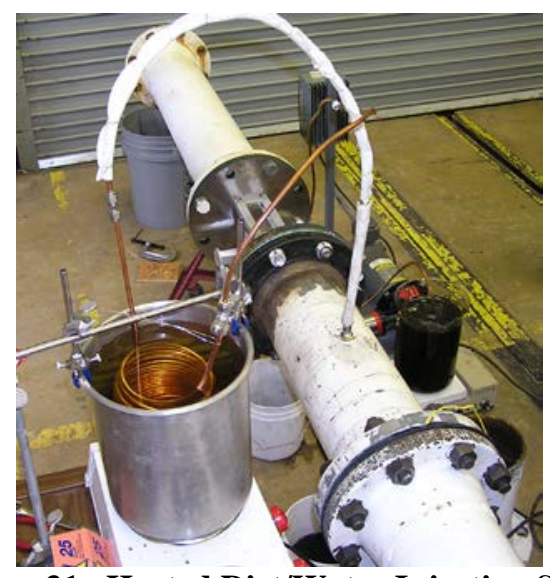

Figure 31. Heated Dirt/Water Injection Setup

Figure 32 and Figure 33 show the results for the redeposit test using deionized water and high-purity deionized water, respectively. The results indicated that dirt could be deposited with both levels of water purity. Although there were minor differences in the deposit patterns as a function of the airflow velocity, the net result of dirt being deposited on the blade was the same for all the tests. Deposit patterns on the back of the blades suggested that the flow structure was better defined for the higher Mach number tests.

These results showed less deposit than the tests with the spray gun and heated air stream; this was likely because the combination of hotter air from the torpedo heater $\left(>400^{\circ} \mathrm{F}\right)$ in combination with better atomization of the carrier fluid provided a more effective means of evaporating the fluid from flow stream. However, both sets of tests suggest the same conclusion; the cleaning fluid does not affect the redeposit of washed dirt particles.

\section{SUMMARY/CONCLUSIONS}

The objective of this project was to provide a thorough experimental evaluation on the effectiveness of online turbine cleaning with various cleaning agents, including high-purity deionized (DI) water.

Dirt was sampled from actual gas turbine axial compressor blades to characterize the composition and consistency of typical blade surface fouling. Samples of dirt were analyzed to determine the chemical composition. A representative dirt formula and blade coating procedure was developed for repeatable testing of blade cleaning and redeposit. Dirt was applied to the compressor blades. The blades were then installed in a wind tunnel with an upstream spray nozzle that simulated compressor operating conditions during online washing. Washing was performed with five different liquids (three grades of water, a commercial compressor cleaning product, and a laundry detergent) to determine the effectiveness of any of the liquids. Once this testing was completed, a similar test setup was utilized to inject a mixture of the formulated fouling dirt with various online cleaning liquids upstream of the blade into the wind tunnel to assess redeposit characteristics. Separate residue experiments were studied to determine the effect of high-purity water versus regular water on fouling dirt. 


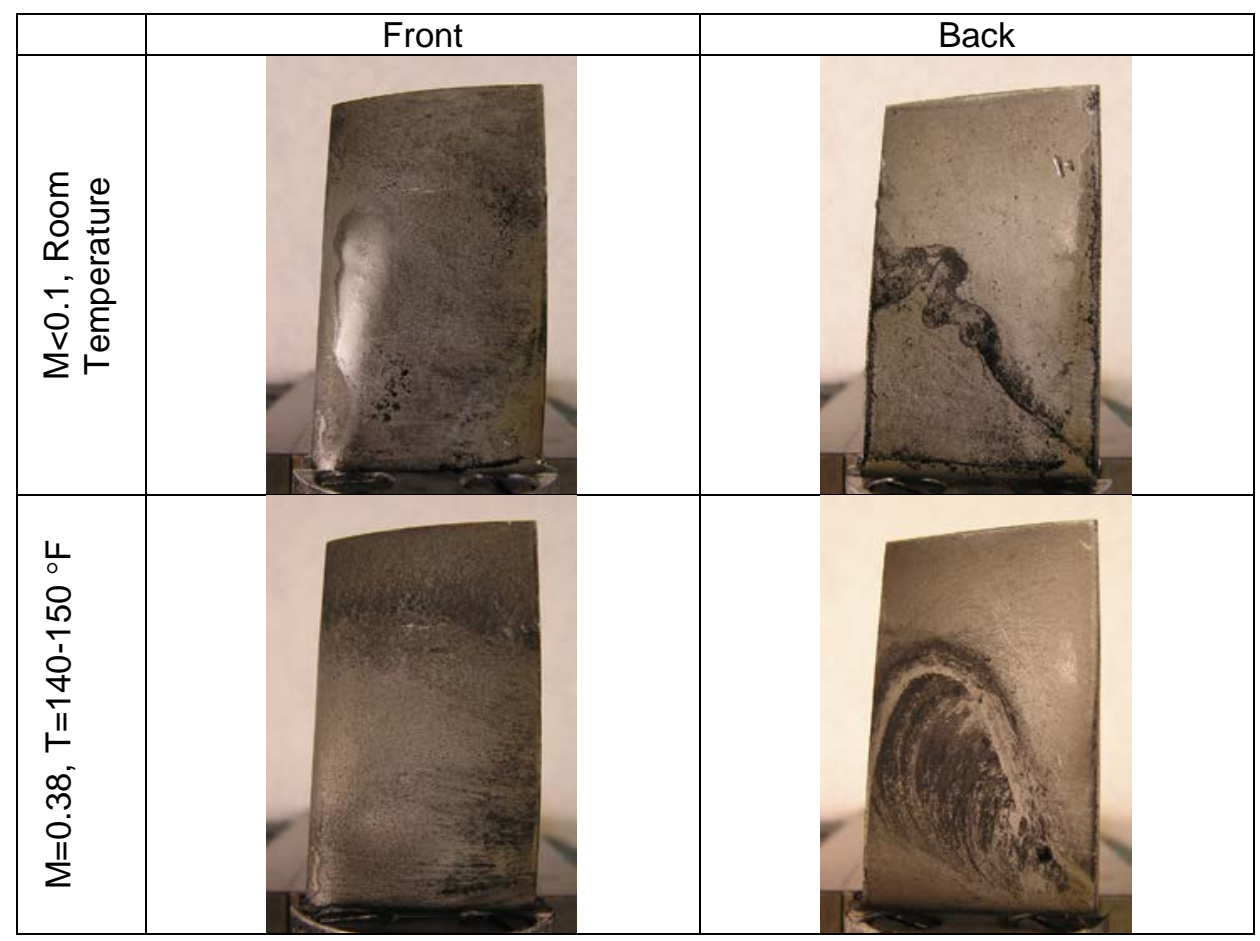

Figure 32. Redeposit Tests with Heated Dirt/DI Water Mixture

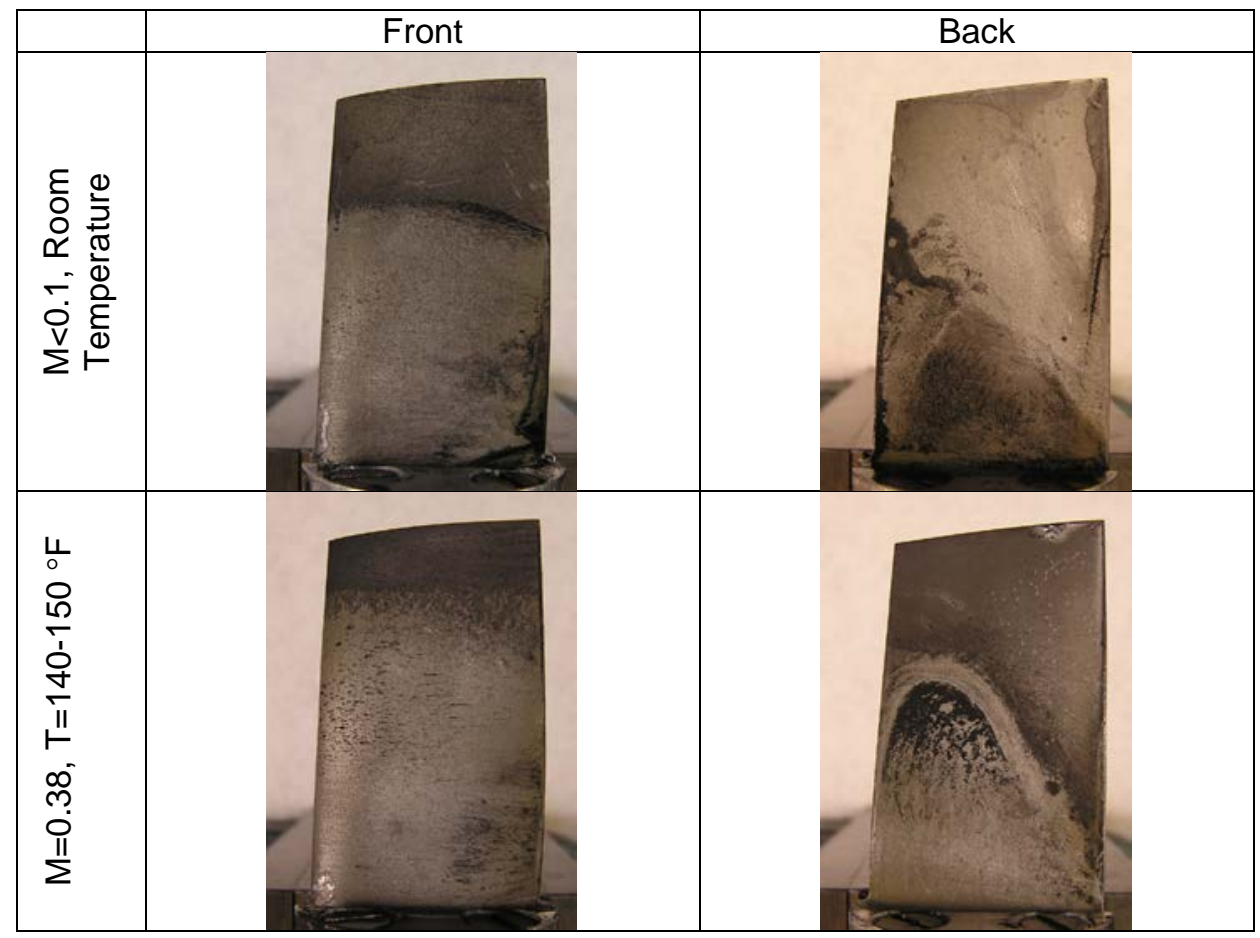

Figure 33. Redeposit Tests with Heated Dirt/High-Purity DI Water Mixture

\section{Conclusions}

Within the parameters/fluids tested in this study, the results indicate that:

1. Spraying cleaning fluid into a flowing air stream is a viable means of cleaning a compressor blade. Each of the fluids was able to clean the test blade at both low and high air velocities and at different blade incident angles. However, for all tested cleaning cases, there was always an area of the blade where some fouling deposits remained.

2. The blade cleaning is primarily a mechanical (droplet impact) function and does not depend on the fluid used for cleaning. Test results show that most of the cleaning occurs shortly after the cleaning fluid is introduced into the flow stream. The type of fluid used did not have a significant impact on the cleaning effectiveness. 
3. Dirt removed from the blades will redeposit in downstream stages as the cleaning fluid is evaporated. Redeposit occurred in flow recirculation zones during the cleaning tests, and heated flow tests demonstrated dirt deposit in the presence of a cleaning fluid. The type of fluid used for cleaning has no effect on the redeposit characteristics of the dirt.

4. Blade erosion was not found to be a significant issue for the short durations that online water-washing is performed. However, uncontrolled water-washing (or overspray) for extended periods of time does result in measureable leading and trailing edge blade erosions.

5. The results suggest that it may be beneficial to the cleaning process to slow the compressor speed or vary the cleaning fluids spray rate while the online wash is being performed.

The results show that most of the cleaning occurs shortly after the cleaning fluid is introduced into the flow stream. Field tests with online water-washing should include tests that vary the cleaning time. The issue of redeposit should be addressed during field testing; if possible, inspection of downstream compressor stages should be used to assess the transport and redeposit of dirt from early to later compressor stages.

The objectives and results of this study can be summarized with the previously posed four questions and answers:

1. Does online cleaning work? Yes, wind tunnel blade test results indicated up to 95 percent removal of blade fouling is possible.

2. Is there any difference between any of the online cleaning liquids? No, there was no clear evidence that any of the liquids or detergent mixes improved the overall blade washing efficiency.

3. Will dirt be removed during online cleaning redeposit once the cleaning liquid has been evaporated? Yes, redeposit tests showed that a significant fraction of the dirt will redeposit on downstream blades. The actual quantity of the redeposit depends strongly on the local flow field and the type of particles that are being carried in the freestream.

4. Do any liquids reduce the redeposit of dirt in the online washing process? No, testing showed that redeposit occurred with all liquids tested, and there was no clear evidence that any mixtures or detergents reduced particle redeposit.

\section{REFERENCES}

Boyce, M. P. and Gonzalez, F., 2005, “A Study of On-Line and Off-Line Turbine Washing to Optimize the Operation of a Gas Turbine," Proceedings of ASME Turbo Expo, Paper No. GT2005-69126, Reno-Tahoe, Nevada.

Elrod, C. E. and Bettner, J. L., 1983, "Experimental Verification of an Endwall Boundary Layer Prediction Method,” Report No. AGRAD CP-351.

Hovland, G. and Antoine, M., 2004, "Economic Optimization of Gas Turbine Compressor Washing,"
Australasian Universities Power Engineering Conference, Brisbane, Australia.

Kurz, R. and Brun, K., 2009, "Degradation Effects on Industrial Gas Turbines,” ASME Journal of Engineering for Gas Turbines and Power, 131, pp. 62401.

Kurz, R. and Brun, K., 2012, "Fouling Mechanisms in Axial Compressors," ASME Journal of Engineering for Gas Turbines and Power, 134, pp. 032401-1-032401-9.

Ogbonnaya, E. A., 2011, "Gas Turbine Performance Optimization Using Compressor Online Water-Washing Technique,” Journal of Engineering, 3, pp. 500-507.

Parker, G. J. and Lee, P., 1972, "Studies of the Deposition of Sub-Micron Particles on Turbine Blades," Proceedings of the Institution of Mechanical Engineers Conference, 186, pp. 519526.

Meher-Homji, C. B., Chaker, M., and Bromley, A. F., 2009, "The Fouling of Axial Flow Compressors-Causes, Effects, Susceptibility, and Sensitivity,” Proceedings of ASME Turbo Expo, Paper No. GT2009-59239, Orlando, Florida.

Mund, F. C. and Pilidis, P., 2004, "A Review of Gas Turbine Online Washing Systems," Proceedings of ASME Turbo Expo, Paper No. GT2004-53224, Vienna, Austria.

Schneider, E., Demircioglu, S., Franco, S., and Therkon, D., 2009, "Analysis of Compressor On-Line Washing to Optimize Gas Turbine Power Plant Performance,” Proceedings of ASME Turbo Expo, Paper No. GT2009-59356, Orlando, Florida.

Syverud, E., Brakke, O., and Bakken, L. E., 2007, "Axial Compressor Deterioration Caused by Saltwater Ingestion," ASME Journal of Turbomachinery, 129, pp. 119-127.

Tarabrin, A. P., Schurovsky, V. A., Bodrov, A. I., and Stalder, J.-P., 1998, "An Analysis of Axial Compressor Fouling and a Blade Cleaning Method," ASME Journal of Turbomachinery, 120(2), pp. 256-261.

Vigueras Zuniga, M. O., 2007, “Analysis of Gas Turbine Compressor Fouling and Washing Online," Ph.D Thesis, Cranfield University, UK. 